\title{
Ann Arbor Pathologic Stage IV
}

National Cancer Institute

\section{Source}

National Cancer Institute. Ann Arbor Pathologic Stage IV. NCI Thesaurus. Code C125479.

A pathologic stage that refers to diffuse extralymphatic involvement by lymphoma. 